The clinical characteristics of the fetal hydantoin syndrome include upturned nose, midfacial hypoplasia, long upper lip, absent cupid's bow, hirsutism of face, back, arms, and legs, nail hypoplasia, hypotonia with delayed motor development, and poor weight gain.

\title{
PHENOBARBITAL-INDUCED DEPRESSION
}

Twenty-eight epileptic children aged six to 16 years were assessed for psychopathology in relation to anticonvulsant monotherapy at the Western Psychiatric Institute and $\mathrm{Clinic}$, Children's Hospital of Pittsburgh and Mercy Hospital, Pittsburgh, PA. Eight patients were treated with phenobarbital, 17 carbamazepine, and three had been wi thdrawn from their anticonvulsant regimen. The phenobarbital treated group showed a higher rate of major depression than did those treated wi th carbamazepine or no anticonvulsant ( $38 \%$ vs $0 \%$ ). The frequency of suicide attempts was similar between groups (13\% vs $12 \%$ ). The phenobarbital treated group had higher scores on the Children's Depression Inventory than did the carbamazepine treated patients. The patients who discontinued phenobarbital therapy recovered fram major depressive disorder whereas those who continued the treatment remained depressed. (Brent DA et al. Phenobarbital treatment and major depressive disorder in children with epilepsy: A naturalistic follow-up. Pediatrics June $1990 ;$ 85:1086-1091).

COMMENT. Despite the relative safety of phenobarbital campared to other anticonvulsants, the increasing number of reports regarding adverse effects on behavior and cognition preclude its use in children whenever possible. Patients should be monitored closely for symptams of an affective disorder and intellectual deterioration, and if signs of depression or regression are detected, a change to an alternative anticonvulsant should be considered. (See PNB January 1990; $\underline{4}: 2$ ).

\section{BENZODIAZEPINE-INDUCED CONGENITAL MALFORMATIONS}

The potential teratogenic properties of benzodiazepine (BZD) intake during early pregnancy were investigated at the Departments of Pediatrics, Pathology and Genetics at Goteborg University, Sweden. Four neonatal diagnoses of congenital malformations known to be characteristic of infants born to mothers with excessive intake of BZD in early pregnancy were present in 25 of 10,646 live born infants $(2.3 / 1,000)$ delivered by mothers living in the city of Gothenburg in 1985 and 1986. The maternal plasma was analyzed in 18 of these cases 1.2 to 1.5 years after the birth of the probands; eight samples (44\%) were BZD positive. Of 60 controls, two maternal blood samples (3\%) were positive for BZD. The difference was highly significant and suggests an association between the congenital malformation and BZD consumed during early gestation. (Laegried L et al. Congenital malformations and maternal consumption of benzodiazepines: A case-control study. Dev Med Child Neur May 1990; $32: 432-441$ ).

COMMENT. The diagnoses considered to be specific for BZD-induced congenital malformations were embryopathy and fetopathy, nervous system malformations, cleft lip and cleft 
palate, congenital malformations of the urinary tract. The authors considered that their findings strengthened the hypothesis that BZD intake during early pregnancy is associated with teratogenicity in man but there is as yet no firm proof of association. Diazepam in early pregnancy should be avoided on the basis of this and other studies which strongly suggest a teratogenic effect of BZD.

\section{NEURO-OPHIHALMOLOGY}

OCULAR SIGNS OF CHIARI MALFORMATION

Twenty-eight patients (14 females and 14 males aged between four and 34 years) with myelomeningocele and Chiari malformations were examined neuro-ophthalmologically at the Karolinska Institute, Huddinge University Hospital, Huddinge, Sweden. The Chiari malformation, determined with MRI, was type $I$ in three patients and type II in 25. All had hydrocephalus; mild in 12, moderate in 13, and marked in three. Shunt procedures had been performed in 20 . Spontaneous or gaze related nystagmus and abnormal optokinetic nystagmus were the most cammon disturbances of ocular motility. Horizontal gaze paresis occurred in 14 patients and vertical gaze limitations in nine, all in upward gaze. Horizontal nystagmus occurred in 17 whereas vertical nystagmus was uncammon and downbeat nystagmus was not observed. Strabismus occurred in 11 patients and esotropia was more cammon than exotropia. No signs of optic atrophy or other changes in the visual pathways were found. (Lennerstrand G, Gallo JE. Neuro-ophthalmological evaluation of patients with myelomeningocele and Chiari malformations. Dev Med Child Neur May 1990; 32:415-422).

COMMENT. Downbeat nystagmus has been considered almost pathognomonic of the Chiari malformation but was absent in the cases in this study. A girl aged ten referred to me recently because of migraine headaches had downbeat nystagmus and a refractive error; the MRI was negative for Chiari malformation and brain stem auditory evoked responses were normal. Lennerstrand $\mathrm{G}$ et al have used the MRI to determine the correlation between disturbances of ocular motility and the degree of hydrocephalus, tactile plate deformity, and dislocation of the cerebellum and medulla oblongata in 28 patients with myelomeningocele. (Dev Med Child Neur May 1990; 32:423-431). All patients had Chiari malformations. Strabismus and spontaneous nystagmus were related to the degree of hydrocephalus and to the amount of lower brain stem deformities. Convergence defects correlated with upper brain stem deformities.

OPTIC NERVE HYPOPLASIA

The pathology, clinical features, and disorders associated with optic nerve hypoplasia in children are reviewed from the Tennent Institute of Ophthalmology, Weston Infirmary, Glasgow, Scotland. A total of 100 references is provided. Histologically a reduced number of optic nerve fibers can be demonstrated in a smaller than normal optic nerve. An overgrowth of retinal pigment epithelium surrounding 